Werte-Bildung interdisziplinär

Band 4

Herausgegeben von

Martina Blasberg-Kuhnke, Eva Gläser, Reinhold Mokrosch,

Susanne Müller-Using und Elisabeth Naurath
Reinhold Mokrosch / Elisabeth Naurath / Michèle Wenger (Hg.)

Antisemitismusprävention in der Grundschule - durch religiöse Bildung

Mit 10 Abbildungen

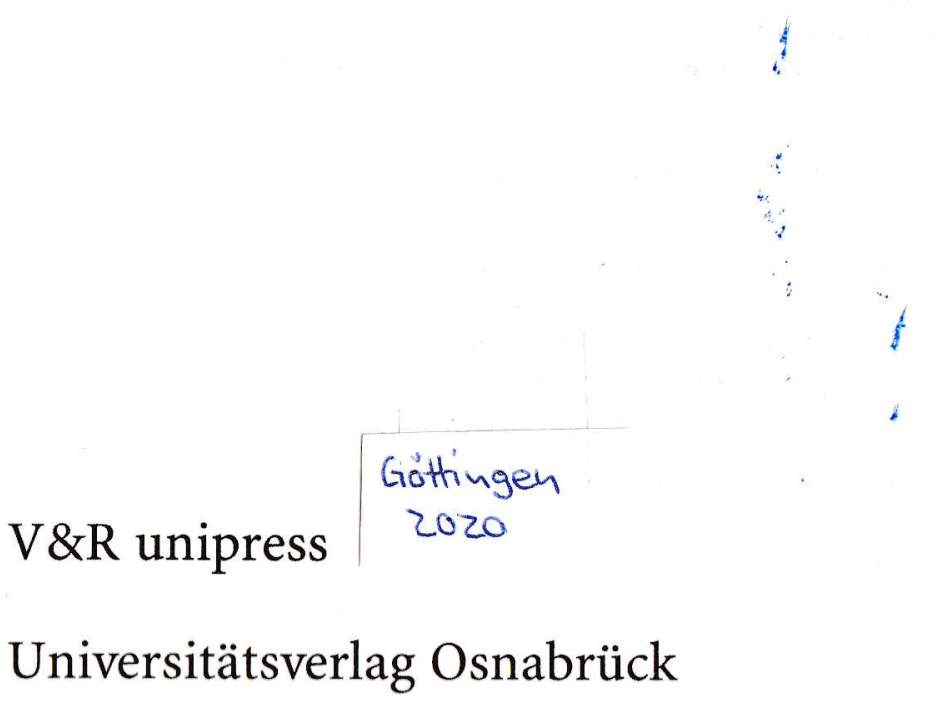


zu fragen und über jüdische Religion, Geschichte und Kultur zu forschen. Und Michèle Wenger, Jahrgang 1991, Zürich, kennt den Zweiten Weltkrieg aus Schilderungen ihrer Großeltern (Zeitzeugen) und arbeitet als Forscherin im Erinnerungslernen.

Wir danken allen Autor*innen der 27 Beiträge dieses Bandes herzlich. Sie repräsentieren eine große Varianz zur »Antisemitismus-Prävention an Grundschulen - durch religiöse Bildung ". Ganz bewusst haben wir die Vielstimmigkeit und zum Teil auch Kontroversität der Meinungen der Autor*innen nicht zensiert oder geglättet, um den Diskurs möglichst lebendig zu halten. Sowohl die unterrichtspraktische wie auch die wissenschaftlich-theoretische Sichtung war uns ein Anliegen. Wir danken besonders Luisa Beck und Jens Beiner aus Augsburg für ihre großartige Arbeit bei Korrekturen und Formatierung.

Wir sind dankbar, wenn dieses Buch dazu beitragen kann, Religionslehrkräfte bei ihrer Aufgabe zur Antisemitismus-Prävention zu unterstützen, damit Grundschulkinder für die jüdische Kultur und Religion aufgeschlossen und vor antisemitischen Vorurteilen bewahrt werden.

Osnabrück, Augsburg, Zürich im Juni 2020

Reinhold Mokrosch, Elisabeth Naurath, Michèle Wenger

\section{Elisabeth Naurath}

\section{Antisemitismus-Prävention im Religionsunterricht der Grundschule - Wagnis, Herausforderung und Ermutigung}

\author{
Die Wunden sind verschorft, \\ aber der Schmerz bleibt \\ auf immer und täglich wieder neu. \\ Erschreckend \\ Herausfordernd \\ Er will uns zeigen, \\ was böse ist \\ und was \\ gut \\ sein kann. \\ Er will uns zeigen \\ was zu hoffen \\ und was zu tun ist. \\ Elisabeth Naurath
}

Der Jahresbericht 2019 zur >Politisch motivierten Kriminalität $`$ (PMK) zeigt, dass antisemitische Straftaten aktuell um rund $13 \%$ gestiegen sind. Eine Entwicklung, die mit großer Sorge beobachtet wird und in allen Bildungsbereichen nach Präventionsmöglichkeiten verlangt. Auch im Kontext religiöser Bildung wird verstärkt gefragt, inwiefern antisemitische als dezidiert judenfeindliche Einstellungen zu sehen sind, ob nicht Synergie-Effekte religiöser und politischer Bildung stärker zu beachten sind und vor allem ob nicht lebensgeschichtlich viel früher mit Präventionsmaßnahmen zu beginnen sei?

"Antisemitismus ist eine bestimmte Wahrnehmung von Juden, die sich als Hass gegenüber Juden ausdrücken kann. Der Antisemitismus richtet sich in Wort oder Tat gegen jüdische oder nichtjüdische Einzelpersonen und/oder deren Eigentum sowie gegen jüdische Gemeindeinstitutionen oder religiöse Einrichtungen. Darüber hinaus 
kann auch der Staat Israel, der dabei als jüdisches Kollektiv verstanden wird, Ziel solcher Angriffe sein. ${ }^{1}$

Während es schon vorchristliche Formen der Judenfeindschaft und des Judenhasses gab, wurde seit dem späten 19. Jahrhundert der Begriff >Antisemitismus meist in rassistischer Perspektive exklusiv abwertend auf das Judentum bezogen. ${ }^{2}$ Antisemitismus wurde zum Oberbegriff antijüdischer Ressentiments, indem letztendlich die "vorgefasste Idee vom Juden als das Wesentliche ${ }^{3}$ allen Differenzierungen entgegengesetzt und auch rückwirkend auf die verschiedenen Facetten von Judenfeindschaft übertragen wurden. Vom ursprünglichen Wortsinn her rekurriert der Begriff `Semit ` auf alle Personen und Personengruppen, die sich nach biblischer Tradition auf Sem, den ältesten Sohn Noahs zurückführen lassen, d.h. im Prinzip die Völker des Nahen Ostens. Dennoch bezieht sich der Begriff `Antisemitismus a ausschließlich auf das grundlegende Phänomen der Judenfeindschaft - nicht aber der Islamfeindlichkeit - in all seinen historischen Formen des Wandels der jeweiligen Begründungszusammenhänge. Auf die hierin liegende Problematik eines »ewigen Antisemitismus « hat beispielsweise schon Hannah Arendt in ihren Schriften hingewiesen, indem eine gewisse historische Unveränderbarkeit suggeriert wird. ${ }^{4}$

Der nur schwer definierbare Neologismus ist hierbei nicht nur im Sinne eines theologischen Antijudaismus als vielmehr wesentlich globaler, allgemeiner und komplexer zu verstehen, nämlich als »Sammelbezeichnung für alle Einstellungen und Verhaltensweisen, die den als Juden wahrgenommenen Einzelpersonen, Gruppen oder Institutionen aufgrund dieser Zugehörigkeit negative Eigenschaften unterstellen. $\aleph^{5}$ Der erste Antisemitismusbericht der Bundesregierung

1 Bei dieser Definition handelt es sich um die Arbeitsdefinition von Antisemitismus der International Holocaust Remembrance Alliance (IHRA) in der von der Deutschen Bundesregierung angenommenen Form, vgl. https://www.auswaertiges-amt.de/de/aussenpolitik/themen/kultur dialog/-/216610; (Stand: 31.08. 2019). Zur Geschichte und Entwicklung dieser Arbeitsdefinition und zu den Schwierigkeiten einer definitorischen Klärung vgl. das entsprechende Kapitel im Bericht des Unabhängigen Arbeitskreises Antisemitismus an den Deutschen Bundestag vom 7.4.2017, S. 23 ff., https://dip21.bundestag.de/dip21/btd/18/119/1811970.pdf; (Stand: vom 7.4.208.2019).

2 Vgl. zum Folgenden auch: Werner Bergmann: Geschichte des Antisemitismus, C.H. Beck Verlag, München 2002, 5. durchgesehene Aufl. 2016,sowie Werner Bergmann: Antisemitismus in öffentlichen Konflikten. Kollektives Lernen in der politischen Kultur der Bundesrepublik 1949-1989, Schriftenreihe des Zentrums für Antisemitismusforschung, Bd. 4, Campus Verlag, Frankfurt a. M./New York 1997.

3 Jean-Paul Sartre, Betrachtungen zur Judenfrage, Zürich 1948, 13.

4 Vgl. z.B. Hannah Arendt, Vor Antisemitismus ist man nur noch auf dem Monde sicher. Beiträge für die deutsch-jüdische Emigrantenzeitung >Aufbau< 1941-1945. München 2000.

5 Deutscher Bundestag (Hg.): Antisemitismus in Deutschland - Erscheinungsformen, Bedingungen, Präventionsansätze. Bericht des unabhängigen Expertenkreises Antisemitismus. Drucksache 17/7700. Berlin 2011, 9. von $2011^{6}$ zeigte bereits, dass antisemitische Einstellungen und Haltungen quer durch die Alters- und Gesellschaftsschichten verlaufen. Bei der drängenden Frage, wie man also diesem flächendeckenden Phänomen vorbeugend begegnen kann, ist besonders evident, dass trotz der breit angelegten Bemühungen einer gesamtgesellschaftlichen Verurteilung von Antisemitismus keine systematische Präventionsarbeit stattgefunden hat und stattfindet. Im Vergleich $\mathrm{zu}$ anderen europäischen Ländern muss mit Robert Sigel konstatiert werden: »Für die Bundesrepublik steht ein Gesamtkonzept noch aus (...) «?

'History doesn't repeat itself, but it does rhyme - so Mark Twain. Geschichte wiederholt sich nicht, aber sie reimt sich. Um diesen Reim quasi als Schema zu durchbrechen, muss sich die Struktur grundlegend ändern, die Struktur des Denkens und damit auch des Redens und Handelns. Wie grundlegend - das zeigt die Geschichte des Antisemitismus in Deutschland, ja sogar in Europa. Hierin liegt die heutige friedenspädagogische Aufgabe angesichts eines nach 80 Jahren erneut aufflammenden Antisemitismus in unserer Gesellschaft. ${ }^{8}$ Einerseits ist dies eine bildungstheoretische Aufgabe für alle schulischen Fächer ${ }^{9}$, andererseits kommt dem Religionsunterricht angesichts der gesellschaftlichen Tradierungsabbrüche zwar quantitativ eine kleiner gewordene, aber aus inhaltlichen Gründen eine besonders wichtige Rolle und Verantwortung zu. Allerdings ist für den deutschen Kontext der Religionsunterricht in der Grundschule ein nahezu weiBer Fleck auf der Landkarte der Präventionsbemühungen. Daher liegt es zu Beginn des Bandes besonders nahe, mit >Guten-Praxisbeispielen` Einblicke in bereits vorhandene und erfolgreiche Modelle zu geben und zur Reflexion, Diskussion und Nachahmung zu ermutigen.

Aus diesem Grund starten wir im 1. Kapitel mit `Gutên-Praxisbeispielen〈, um Einblicke in bereits vorhandene und erfolgreiche Modelle zu geben. Zunächst wird das niedersächsische Kooperationsprojekt ) Judentum begreifen` (Elisabeth Naurath, Heide Rosenow, Aloys Lögering, Reinhold Mokrosch) vorgestellt. Es lebt von der Begegnung mit Menschen jüdischen Glaub̧ens, die in Projekttagen an Grundschulen anhand von Gesprächen, Präsentationen, Workshops, Film und Theater ihre Glaubens- und Festtraditionen vorstellen und mit den Kindern ins Gespräch kommen. Auch das Thema Shoa wird hierbei nicht ausgeklammert,

6 Ebd.

7 Robert Sigel, Das Thema >Antisemitismus in der schulischen Bildung. In: Bayerische Landeszentrale für politische Bildungsarbeit https://www.blz.bayern.de/meldung/das-thema-ran tisemitismusr-in-der-schulischen-bildung.html; (Stand: 06.02.20).

8 Vgl. hierzu auch: Lipstadt, Deborah: Der neue Antisemitismus. München 2018; Blume, Michael: Warum der Antisemitismus uns alle bedroht. Wie die neuen Medien alte Verschwörungstheorien befeuern. Ostfildern 2019.

9 Vgl. Matthes, Eva/ Meilhammer, Elisabeth (Hg.): Holocaust Education im 21. Jahrhundert. Kempten 2015. 
sondern mit der Einladung einer Zeitzeugin für die Grundschulkinder vergegenwärtigt.

Den Gedanken des Begegnungslernens aufgreifend ist in internationaler Perspektive spannend, wie Michéle Wenger das seit 2002 bestehende Projekt sLikrat aus der Schweiz vorstellt, das mittlerweile auch in Österreich und Deutschland übernommen wurde. Das Dialogprojekt Likrat (das hebräische Wort für saufeinander zugehen`) sendet ausgebildete Likratinos und Likratinas mit dem Ziel der Information, Begegnung, Mediation und Bildung auch in Schulen, um antisemitischen Einstellungen vorzubeugen bzw. diese abzubauen. Gerade mit Blick auf die religionsbezogene Bildung an Volksschulen ist diese Initiative hinsichtlich der Kompetenzziele nicht nur anschlussfähig, sondern zukunftsweisend.

Dies gilt ebenso für die Arbeit des Vereins »ZWEITZEUGEN e.V.«, der sich aus dem Studienprojekt Heimatsucher e.V. entwickelt hat und die Katharina MüllerSpirawski und Vanessa Eisenhardt in ihrem Artikel beschreiben. Seit der Gründung mit dem Ziel, die Lebensgeschichten Shoa-Überlebender auf ehrenamtlicher Basis in die Bildungseinrichtungen zu bringen, versetzen sie sich als Zweitzeug*innen in die Biographien von Zeitzeug*innen, um Schüler*innen zu sensibilisieren, die Lebensgeschichten lebendig zu erhalten und ihnen zu helfen, auch selbst friedenspädagogische Verantwortung zu übernehmen.

Das Präventionsziel einer Förderung der Kenntnisse und des Verstehens der jüdischen Religion verfolgt auch das Lehrhaus für Kinder der Alten Synagoge Essen, das Martina Strehlen für die 3. und 4. Grundschulklasse vorstellt. Gerade dieser historische und mit einem Bildungsprogramm neu konzipierte Lern- und Erfahrungsort bietet die Möglichkeit, dass Kinder dem Judentum in seiner Tradition und in seiner Lebendigkeit begegnen. Insofern steht dieses , GutePraxisbeispiel nicht nur als Modell für Synagogenpädagogik, sondern auch für die Chance interreligiösen Dialogs.

Eine Klärung der Frage, welche Phänomene und Einstellungen als Antisemitismus anzusehen sind, um von hier aus Präventionsstrategien zu entwickeln, ist besonders problematisch. Bei Sichtung der Literatur fällt auf, dass die Heterogenität der Begriffsklärungen zum Antisemitismus auf die Komplexität des Phänomens verweist. Für diesen Band sollen daher im 2. Kapitel einleitend begrifflich-historische Klärungen eine für Lehrkräfte grundlegende Orientierung bieten. Der im christlich-jüdischen Dialog seit vielen Jahrzehnten engagierte Träger der Buber-Rosenzweig-Medaille Hanspeter Heinz erläutert die klassisch gewordenen Typen des Antisemitismus, indem er sie historisch von der Antike bis heute verortet. In einem weiteren Schritt zeigt er die Wende im christlichjüdischen Verhältnis mit dem Zweiten Vatikanischen Konzil auf und betont die Notwendigkeit einer Neuinterpretation antijüdischer Aussagen von christlicher Seite. Schließlich beschreibt er Formen latenten Antisemitismus in Kirche und
Gesellschaft und plädiert dafür, Antisemitismus nicht zum zentralen Thema im Blick auf Juden werden zu lassen. Während Hanspeter Heinz die kirchliche Präventionsarbeit fokussiert, sieht Ludwig Spaenle, der seit 2018 Antisemitismusbeauftragter der Bayerischen Staatsregierung ist, den staatlich-schulischen Bereich in seiner wertebildenden Aufgabe - auch zur Antisemitismus-Prävention - herausgefordert. In seinem Beitrag betont er, dass Präventionsarbeit lebensgeschichtlich möglichst früh ansetzen und daher stärker als bisher auch die Grundschule in die Verantwortung nehmen sollte. Kernelement der Wertevermittlung in der Grundschule sei auch Antisemitismus-Prävention. Sie sei zu stärken, indem deutlicher als bisher Informationen über jüdisches Leben in seiner Vielfalt an die Grundschulkinder vermittelt werden sollten.

Doch welche Möglichkeiten bietet religiöse Bildung, um AntisemitismusPrävention im Religionsunterricht der Grundschule zu fördern? Im 3. Kapitel sollen daher religiöse (christliche und muslimische) wie auch interreligiöse Ansätze zur Sprache kommen, die zukunftsweisende Chancen ausloten. Hierbei ist wichtig zu sehen, dass eine dezidierte Antisemitismus-Prävention für religiöse Bildungsprozesse mit Kindern bislang zu wenig im Blick war. Aus diesem Grund steht am Beginn dieses Kapitels der Beitrag von Elisabeth Naurath, der entwicklungspsychologische Bedingungen der Vorurteilsprävention reflektiert, da Antisemitismus auch als religiöses Vorurteil gesehen werden kann und insofern gerade für den Religionsunterricht evident ist. Inwiefern gerade das Alter der 3./4.-Klässler zur Vorurteilsprävention günstig ist und religionspädagogische Interventionsmöglichkeiten bietet, wird anhand von Praxisbeispielen auch zum interreligiösen Lernen aufgezeigt. Religionspädagogischer Hintergrund dieser im dritten Hauptteil aufgezeigten Konzepte ist letztich eine Verortung im Konzert der Friedenspädagogik. So zeigt Reinhold Mokrosch in seinem Artikel, dass Friedenserziehung und Antisemitismus-Prävention ein Geschwisterpaar sind. Ausgehend von einer Klärung der Begrifflichkeitèn wird gezeigt, dass eine Entwicklung zur Friedensfähigkeit mit einer Entwicklung zum Widerstand gegen Antisemitismus Hand in Hand gehen kann und soll, auch wenn dies von verschiedenen Einflussfaktoren wie Intelligenz, Moral, Sozialverhalten und Persönlichkeitsstrukturen abhängig ist. Der Beitrag religiöser Ffiedenserziehung liege jedoch gerade darin, zu betonen, dass alle Menschen als Ģottes Geschöpfe angesehen werden sollten und somit kein Raum für Antisemitismus gegeben wird.

In einem weiteren Schritt geht es um die Bedeutung religiöser Bildung für eine Antisemitismus-Prävention in der Grundschule. Georg Langenhorst stellt in seinem Beitrag das trialogische Lernen als zukunftweisende Option auch zur Antisemitismus-Prävention in den Vordergrund, da der Fokus >Abraham ‘ auf den gemeinsamen Ursprung von Judentum, Christentum und Islam in den letzten 30 Jahren zu den erfolgreichsten theologiegeschichtlichen Entwicklungen 
zähle. Hierbei wirke das trialogische Lernen als Grundprinzip christlichen Denkens und sei damit im Sinne gegenseitiger Wertschätzung als Gegenkraft gegen antisemitische Tendenzen zu verstehen. Für Langenhorst kann es eine optimale Grundlage für interreligiöses Lernen sein, die plurale Welt direkt als Kind kennen zu lernen. Dies geschieht durch Lernen in einer heterogenen Gruppe mit jüdischen, muslimischen und christlichen Kindern, wie dies beispielsweise in der »Drei-Religionen-Grundschule « in Osnabrück der Fall ist. Aber auch der Einsatz von Medien wie der Kindertora, die zu einem Vergleich mit Kinderbibel und Kinderkoran anregen, kann der Austausch über gemeinsame und verschiedene Sprachstile, Gottesvorstellungen u.a. in ein trialogisches Gespräch münden. So gibt es viele Wege im Miteinander der Glaubenden der abrahamischen Religionen schon im Grundschulalter Antisemitismus-Prävention zu leisten.

Dass das Recht jedes Kindes auf religiöse Bildung auch AntisemitismusPrävention umfassen soll, mag wünschenswert sein, ist aber aufgrund der Komplexität dieses Anspruchs auch problematisch: Thomas Schlag beschäftigt sich daher in seinem Beitrag mit entwicklungspsychologischen, (religions)pädagogischen und politikdidaktischen Dimensionen einer für die Grundschule geeigneten Subjektorientierung, die bildungstheoretischen Grundsätzen genügt und zugleich Antisemitismus-Prävention mit dem Grundgedanken des Menschenrechtsbezug religiöser Bildung verbindet. Dieser bereichernde und weiterführende Blick über die deutsche Grenze hinaus ins Nachbarland Schweiz wird mit dem Beitrag von Michèle Wenger fortgesetzt, denn hinsichtlich der religionsdidaktischen Chancen einer Antisemitismus-Prävention in der Grundschule ist der schweizerische Kontext, in dem einerseits auch antisemitische Tendenzen in der Bevölkerung auszumachen sind und man andererseits aufgrund der Neutralität während der Zeit des Nationalsozialismus einen weniger belasteten Zugang zum Thema Holocaust findet. In ihrem Beitrag gibt sie einen Einblick in die Ergebnisse der REMEMBER-Studie, genauer in Antworten von $125 \mathrm{Lehr}$ personen der Schweiz. Dabei wird klar, dass die Lehrpersonen Antisemitismus und den Holocaust für Themen des Religionsunterrichts halten und mit ihnen auch zentrale Aufgaben zur Antisemitismus-Prävention erfüllt werden können: Dazu zählt das Erkennen totalitärer Tendenzen in der heutigen Gesellschaft, die Wissensvermittlung über die Geschichte des Holocaust und hierauf aufbauend die Förderung von gesellschaftlichem Verantwortungsbewusstsein. Als Ausblick fordert die Autorin Unterstützung für die Lehrkräfte in Form von Fortbildungsmöglichkeiten, aber auch Lehrmitteln zur Thematik.

Aus muslimischer Sicht fragt Michael Kiefer nach Antisemitismus-Prävention als Aufgabe interkultureller und interreligiöser Bildung von Kindern, wobei in der Altersgruppe von sechs bis zwölf Jahren bisher keinerlei Studien über antisemitische Haltungen durchgeführt wurden. Als Problemfelder, die möglicher- weise auch antisemitische Haltungen bei Kindern auslösen können, beschreibt er die einseitige Rezeption des Nahostkonfliktes, eine Kolportage antisemitischer Narrationen über einen langen Zeitraum und außerdem eine fehlende zivilgesellschaftliche Auseinandersetzung mit antisemitischen Einstellungen. Grundlegend widmet er sich der Frage, was Muslime zur Bekämpfung antisemitischer Haltungen tun können? Neben dem familiären Umfeld liegen seines Erachtens zukunftsweisende Präventionsmaßnahmen in gemeindlicher Kinder- und Jugendarbeit, in Eltern- und Familienarbeit, Koranunterricht und Begegnungsarbeit. Vom Islamischen Religionsunterricht fordert er, reflektierte Zugänge zu den Quellen zu ermöglichen, Perspektiven für eine Verständigungsbereitschaft $\mathrm{zu}$ öffnen und interreligiösen Dialog zu fördern. Als Grundlage hierfür sieht er Handlungsbedarf im Bereich der Aus- und Weiterbildung von Religionslehrkräften - als eine zukunftsweisende Perspektive.

Deutlich zeigt sich, dass die Thematisierung der Shoa hinsichtlich der Entwicklung von Strategien zur Antisemitismus-Prävention nicht ausgeklammert werden kann - schon gar nicht im Unterricht an deutschen Schulen. So widmet sich das 4. Kapitel einer intensiven Diskussion um die Möglichkeiten einer Didaktik nach der Shoa im Religionsunterricht der Grundschule. Hierzu sollen zunächst Kriterien erstellt werden, die den durchaus kritischen Diskurs aufnehmen und zugleich nach einer kindgerechten Didaktik fragen. In ihrem Beitrag geht Elisabeth Naurath davon aus, dass Grundschulkinder - gerade weil sie erwiesenermaßen im Alltag in den Familien oder über Medien Hinweise zum Holocaust aufschnappen - nicht vor der Begegnung mit der Thematik an sich geschützt werden können - wohl aber vor einer unbegleiteten Konfrontation. Vielmehr ist es wichtig, die Kinder mit ihren Fragen ernst zu nehmen, die zeitliche Distanz zum Holocaust so zu berücksichtigen, dass die Lernenden selbst Bezugspunkte finden können. Eine kindgerechte Didaktik hat selbstverständlich entwicklungspsychologische Bedingungen zu beachten und auf dieser Basis weniger historische Kenntnisse als vielmehr eine Förderung prosozialer Kompetenzen anzuvisieren. Wichtigstes Ziel bleibt bei allem, Traumatisierungen zu verhindern und sensibel, subjektorientiert mit einer gut réflektièrten, für Kinder angemessenen Methodik zu unterrichten.

$\mathrm{Ob}$ und wie dies in der Praxis auch gelingt, ist bislang für den Religionsunterricht empirisch unerforscht. Insofern ist eine erste, nicht repräsentative Befragung von Lehrkräften hilfreich und weiterführend: Reinhold Mokrosch zeigt in der Auswertung seines Fragebogen-geleiteten Gesprächs mit Lehrkräften verschiedener Schularten, dass die Befragten der Meinung sind, dass Antisemitismus-Prävention in der Grundschule ansetzen müsse. Die Vorschläge, wie man dies allerdings in den Unterricht einbringen würde, waren sehr unterschiedlich. Wichtig war dabei das Thematisieren von Einzelbiografien, jedoch ohne die Kinder emotional, moralisch oder religiös zu überfordern. Die meisten Lehr- 
kräfte waren davon überzeugt, dass durch Antisemitismus-Prävention im Unterricht die Schüler*innen empathiefähiger geworden seien.

Auch Reinhold Boschki plädiert in seinem Beitrag für eine Sensibilisierung von Kindern für Antisemitismus im Religionsunterricht der Grundschule, indem er die Dimension des Erinnerungslernens beleuchtet und die gegenseitige Ergänzung von emotionaler und kognitiver Dimension des Erinnerns betont. $\mathrm{Da}$ religiöse Bildung genuin anamnetische Strukturen aufweist, die sowohl individuelles wie auch kulturelles Erinnern umfasst, spielt auch das Erinnern des Holocaust eine evidente Rolle. Hierzu hat es sich die Forschungsgruppe REMEMBER zur Aufgabe gemacht, im Rahmen einer Bestandsaufnahme auch Religionslehrkräfte an Grundschulen zu befragen. Deutlich zeigt sich, dass sowohl Erinnern als auch Erzählen als geeignete Lernwege ineinandergreifend die Empathiefähigkeit bei Schüler*innen erhöhen, wobei immer die Betonung des lebendigen Judentums im Blick bleiben sollte.

Zur eingehenderen Klärung der unterrichtspraktischen Zugänge ist der Blick in die Praxis von Lehrkräften unabdingbar, so dass Tina Raddatz-Schick ihre Erfahrungen mit Bezug zum niedersächsischen Kerncurriculum und Bildungsauftrag für den Religionsunterricht hinsichtlich einer intendierten Antisemitismus-Prävention beschreibt. Obwohl im Religionsunterricht Themenbereiche von Neid, Vorurteil und Ausgrenzung bearbeitet werden, bemängelt sie, dass Antisemitismus-Prävention nur indirekt vorkomme. Bei der praktischen Umsetzung stellt sie fest, dass die Lernenden sehr wissbegierig seien und auch Präkonzepte bestünden. Es sei hierbei immer zu bedenken, wie viel den Kindern schon zuzumuten ist und wo Grenzen liegen. Auch Georg Wagensommer beschreibt in seinem Artikel Möglichkeiten zur Antisemitismus-Prävention in der Grundschule ausgehend von einer Didaktik der Shoa, indem er den Weg wählt, Ergebnisse verschiedener empirischer Studien zur Holocaust-Didaktik zugrunde zu legen. Klar zeigt sich, dass das Judentum kein Teil der Lebenswelt der Schüler*innen ist und sie vorrangig über die Medien Kenntnisse generieren. Es wird deutlich, dass auch Kinder schon früh und auch außerhalb von Schule mit der Thematik des Nationalsozialismus in Kontakt kommen, wobei ein "Hitlerzentrismus« auffällt. Jüdinnen und Juden werden mehrheitlich in einer Opferperspektive wahrgenommen, wobei Opfer, Täter und Zuschauer eher entindividualisiert und anonymisiert werden. Wagensommer sieht hier die Lehrkraft vor der großen Herausforderung, die Schüler*innen sowohl kognitiv als auch emotional im Rahmen religiöser Bildungsprozesse abzuholen, um sowohl Multiperspektivität wie auch Gegenwartsbezüge zu generieren.

Reinhold Mokrosch beschreibt konkretisierend, wie er eine Unterrichtseinheit über "Janusz Korczak und seine Waisenkinder im Warschauer Ghetto" in einem 3. Schuljahr miterlebt hat; und er untersucht, ob die 9-Jährigen mit den gleichen Gefühlsstrukturen, mit denen sie alltägliche Leiderfahrungen verar- beiten, auch auf das Leiden der Waisenkinder in der Shoa reagieren. Er beobachtet große Differenzen. Und er befragt außerdem 12/13-Jährige, wie sie mit religiösen Antworten auf die Frage 'Warum gibt es Leid?^ umgehen - dies auch aktuell am Beispiel der unter Corona Leidenden. Die Befragten lehnten, wie er feststellt, religiöse Antworten durchweg ab, obwohl sie sich zum Teil als religiös konnotierten.

Abschließend und zugleich für die Praxis eröffnend, widmet sich das 5. Kapitel religionsdidaktischen Impulsen einer Antisemitismus-Prävention für die Praxis. Joachim Willems und Ariane Dihle betonen die identitätsfördernde Dimension religiöser und konfessioneller Bildung, indem sie Religionsbücher für das 3./4. und 5./6. Schuljahr daraufhin untersuchen, ob kategoriale - und daher nicht selten diskriminierende - Zuschreibungen zum Judentum im Sinne eines Gegenentwurfs zum Christentum in den Schulbüchern zu finden sind. Am Modell des `Othering und der Ausblendung multipler Identitäten lassen sich solche essentiellen Zuschreibungen als folgenschwer aufzeigen. Daher plädieren beide Autoren für eine Verdeutlichung der Verwurzelung christlicher Theologie im Judentum, für Differenzsensibilität und Rekategorisierung, z. B. mit Hilfe von Begegnungslernen und literarischem Lernen. Den Faden bezüglich Lehrplänen und Unterrichtsmaterialien aufgreifend setzt die an der Universität Wien (Österreich) tätige Religionspädagogin Julia Spichal den kritischen Blick in die Lehrpläne und Schulbücher der Primarstufe fort und verweist hierzu auf ihre Monografie "Vorurteile gegen Juden im christlichen Religionsunterricht", aus der der vorliegende Artikel einige Ergebnisse von Analysen aufnimmt. Schon Analysen seit den 1980er Jahren hatten betont, dass bei einigen Themen die christliche Identität auf Kosten des Judentums veranschałlicht werde. Wie Spichal analysiert, gab es seit diesen Analysen zwar Verbesserungen, jedoch wurden verschiedene Bereiche dennoch nicht berichtigt; dies gilt insbesondere, wenn keine Verweise darauf zu finden sind, dass das Alte Testament auch die Heilige Schrift des gegenwärtigen Judentums ist, oder wenn Nächsten- und Feindesliebe, das Vaterunser, die Bergpredigt oder auch die Zehn Gebote ohne Rekurs zum Judentum dargestellt werden. Wichtig sei demgegenüber, schon in der Primarstufe via Lehrbücher und Lehrplänen Verbindungslinien zwischen Judentum und Christentum zu betonen, um möglichst früh antijüdischen Vorurteilen entgegenzuwirken.

Ein Schlüssel hierzu ist in der jüdischen Gegenwartskultur zu sehen, die Roland Biewald in konkrete Unterrichtsbausteine für die höheren Grundschulklassen 3 und 4 so übersetzt, dass sie sich zur Prävention von antisemitischen Einstellungen eignen. Biewald setzt darauf, Zugänge dort zu suchen, wo die Kinder mit Vorurteilen, Ausgrenzungen, Beschimpfungen und Beleidigungen in Berührung kommen: Indem die Schüler*innen Mechanismen der Ausgrenzung reflektieren, diese mit einer biblischen Geschichte verbinden und spielerisch 
Verhaltensstrategien gegenüber ausgegrenzten Menschen einüben, wird als nächster Schritt im Unterricht die Begegnung mit einer biographischen Erzählung mit einem altersgleichen Mädchen (Lebensgeschichte von Henny Brenner als Beispiel von Verfolgung in der Shoa) geführt, um sich mit den Gründen auseinanderzusetzen, warum gerade Juden ausgegrenzt wurden und werden. Abschließend soll dann ein Einblick in jüdische Gegenwartskultur ermöglicht werden.

Vertieft wird diese Chance zur Antisemitismus-Prävention via literarischen Lernens in dem zweiten Beitrag von Georg Langenhorst: gegenwärtige jüdische Kinder- und Jugendliteratur eignet sich hervorragend, in interkultureller und religionspädagogischer Perspektive dezidiert religiöse Facetten heutigen Judentums ansprechend und interessant zu verlebendigen, um subjektive Perspektiven quasi spielerisch verfremdet $>$ von innen $>\mathrm{zu}$ erlesen. Gerade diese Texte der deutschen Gegenwartsliteratur eignen sich zur Antisemitismus-Prävention, da sie nicht vorrangig mit Blick auf die Shoa vergangenheitsbewältigend ausgerichtet sind.

Im folgenden Beitrag präsentiert Benigna Schönhagen die Augsburger Synagoge als außerschulischen Lernort, um ebenfalls lebendiges Judentum den Schüler*innen nahe zu bringen. An diesem Lernort soll besonders die Förderung interkultureller Bildung im Vordergrund stehen, die hier durch den Erwerb von religiösem Wissen geschieht. Die Chancen und Grenzen für die AntisemitismusPrävention sowohl dieses Kooperationsprojektes von Schule und Museum, als auch von Synagogenführungen werden in diesem Beitrag deutlich. Ziel des Projektes ist stets den Schüler*innen positive Erfahrungen beim Kennenlernen des Judentums und dem ersten Austausch darüber zu ermöglichen. Diesen Faden aufgreifend beschreibt Heide Rosenow in ihrem Artikel biographiebezogene Lernformen der Antisemitismus-Prävention aus christlicher Perspektive. In ihrem Beitrag will sie als erfahrene Grundschullehrkraft und Seminarleiterin exemplarisch anhand der Biographie der Holocaustüberlebenden Erna de Vries Wege aufzeigen, welche die Kinder im Grundschul-Religionsunterricht gegen Judenfeindlichkeit sensibilisieren. Rosenow rät hierbei auch zu lokalen Bezügen, da Kinder nicht selten mit Vorwissen Anknüpfungspunkte an außerschulische Lernorte mitbringen, die kontextbezogen und wirkungsvoll akzentuiert werden können.

Selcen Güzel wendet sich in ihrem Beitrag "Antisemitismus-Prävention im islamischen Religionsunterricht mit Praxisbeispielen« einem sehr wichtigen Thema zu: da sowohl Judentum wie auch Christentum Themen im Lehrplan in der dritten und vierten Jahrgangsstufe sind, kann und soll hier auch der Komplex friedliches Zusammenleben, Fremden- und Judenfeindlichkeit thematisiert werden kann. Äußerst ansprechend: Der Blick in Unterrichtspraxis einer IslamStunde und die reflektierte unterrichtspraktische Reaktion auf eine judenfeind- liche Äußerung einer Schülerin (beeinflusst durch das Elternhaus). Der erste Baustein beinhaltet eine Visualisierung des Vorwissens der Schüler*innen, der zweite Baustein ein Brainstorming zum Vorwissen und einer Sensibilisierung durch Selbstreflexion. Von der hierdurch entstandenen Diskussion unter den Lernenden leitet die Lehrkraft auf das Thema »udentum als eine von Gott offenbarte Religion« über, wozu die Vermittlung von Basiswissen den dritten Baustein darstellt. Als vierten Baustein bringt Güzel die Differenzierung zwischen religiösen und politischen Themen im Kontext des Judentums ein, um schließlich gesellschaftsrelevante Reflexionsfähigkeit der Schüler*innen zu fördern. Gerade in dieser Unterrichtsphase ist es der Lehrkraft wichtig, dass die Schüler*innen kritisch und tolerant mit kontroversen Meinungen ihrer Mitschüler*innen umgehen.

Last but noch least bedarf es eines zukunftsweisenden Blicks in die Unterrichtspraxis der Grundschule, die perspektivisch nur mittels der Lehramtsausund fortbildung eine wachsende Sensibilisierung zur Antisemitismus-Prävention erreichen kann. Jasmin Kriesten zeigt auf der Basis theologischer und kirchlicher Grundannahmen Desiderate hinsichtlich der Lehramtsbildung für Religionslehrkräfte auf und erarbeitet inhaltlich begründete Kompetenzbereiche, die vorrangig Pluralitätsfähigkeit und Friedensbildung für den Religionsunterricht stark machen. An einem konkreten Seminarbeispiel zeigt sie schließlich, wie gemeinsam mit Studierenden für die Praxis erarbeitete didaktische Unterrichtsbausteine aussehen könnten, die religiöse Bildung mit dem Ziel der Antisemitismus-Prävention konstruktiv verbinden. Der Bogen schließt sich mit unterrichtspraktischen Vorschlägen zum Coverbild dieses Band von Rainer Oberhänsli-Widmer von 1997, indem Michèle Wenger das Thema Hoffnung als bilddidaktischen Impuls in verschiedenen methodischen Zugängen aufgreift und kindgerecht umsetzt. 